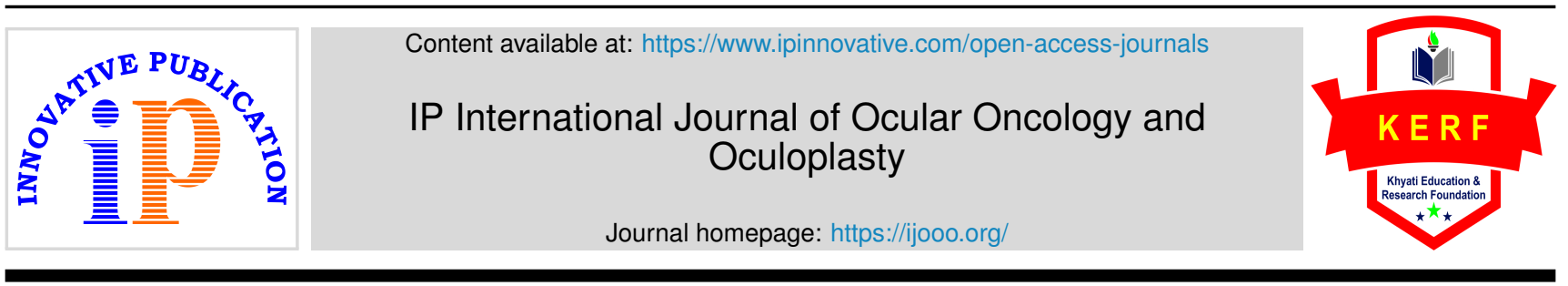

Original Research Article

\title{
A Comparative study of post-operative results with different grades of diabetic retinopathy after phacoemulsification and small incision cataract surgery (SICS) [with various types of IOLs]
}

\author{
Aparajita Chaudhary ${ }^{1, *}$, Smriti Kumari ${ }^{1}$ \\ ${ }^{1}$ Dept. of Ophthalmology, M.L.N.Medical College, Prayagraj, Uttar Pradesh, India
}

\section{A R T I C L E I N F O}

\section{Article history:}

Received 24-12-2020

Accepted 14-01-2021

Available online 23-01-2021

\section{Keywords:}

Diabetic Retinopathy

Phacoemulsification

\begin{abstract}
A B S T R A C T
Purpose: The objective of this study is to look for post-operative complications after cataract surgery in patients with Diabetic Retinopathy.

Materials and Methods : Out of sixty patients, we took fifteen patients each from BDR, Pre-PDR, PDR and DR with maculopathy respectively and patients underwent SICS and phacoemulsification with implantation of IOLs. We looked for post-operative inflammation (aqueous flare, cells, pigment deposition on optic of IOL), incidence of posterior capsular opacification, progression of underlying diabetic retinopathy, incidence of cystoid macular edema and visual outcome.

Result: We observed that visual acuity after cataract surgery improves to $>6 / 12$ in $22 \%$. No patient with BDR grade developed post-0perative uveitis. Maximum inflammation post-operatively was with PDR and pre-PDR was $26 \%$. Cystoid macular edema developed in 26 (55\%) patients.

Conclusion: Visual outcome improves in majority of eyes and was not dependent upon the surgical technique employed or the type of IOL implanted. Post- operative uveitis was maximum with PDR. Majority of patients with PDR developed cystoid macular edema. Progression of retinopathy and maculopathy represented the natural history of disease not upon the surgical technique employed.
\end{abstract}

(C) This is an open access article distributed under the terms of the Creative Commons Attribution License (https://creativecommons.org/licenses/by/4.0/) which permits unrestricted use, distribution, and reproduction in any medium, provided the original author and source are credited.

\section{Introduction}

With introduction of insulin as therapeutic management for DM it was no more a fatal disease for young diabetic patients. The medical care tough shifted upon managing the complications mainly vascular include cardiac and renal disease and diabetic retinopathy.

Cataract occur commonly in diabetic patient for which surgical intervention is required both for visual rehabilitation and visualization of fundus. Development of cataract and retinopathy are more frequent in diabetics depends both on age of the patient and duration of disease.

Patients without macular edema with minimal non proliferative retinopathy have the best chance of attaining post-operative visual outcome of $20 / 40$ or better

\footnotetext{
* Corresponding author.

E-mail address: dr.aparajita.ald@gmail.com (A. Chaudhary).
}

Cataract surgery in diabetics may have more complication than general population including inflammation, CSME, increased risk of progression of retinopathy and anterior segment neovascularization. Intraoperative more miosis, high chance of posterior capsule rupture, elevation of intraocular pressure and even breakdown of blood aqueous barrier in advanced retinopathy cases.

Intraoperative trauma and immunological reaction lead to breakdown of blood-aqueous barrier which causes protein leakage into aqueous humor. The type of surgery and implantation of IOL influences the degree of blood aqueous barrier breakdown and post-operative inflammation. Phacoemulsification with IOL implantation in bag causes less inflammation and breakdown of blood aqueous barrier compared to other surgical procedures. 
Even with SICS or heparin surfaced modified IOLs postoperative inflammation was reduced.

In this prospective study postoperative results after phacoemulsification with implantation of foldable acrylic hydrophobic IOL, conventional and SICS with modified or non-surface modified IOL, in patients with different grades of DR is compared.

Post-operatively looked for inflammation, posterior capsular opacification, progression of DR, visual outcome, cystoid macular edema.

\section{Materials and Methods}

This study was conducted at department of ophthalmology at Regional Institute of ophthalmology, M.D. Eye hospital, Dr. Katju road, Nakhaskona, MLN Medical College, Prayagraj. Total sixty patients were included in the study.

Study was conducted after approval from Institutional review board. All procedures were carried out after obtaining consent from patient. Patients were categorized as BDR, Pre PDR, PDR and DR with maculopathy without other systemic or ocular diseases and fifteen patients was taken in each category respectively. Other non-operated eye was taken as control. These patients underwent cataract extraction by phacoemulsification or conventional or small incision cataract surgery (SICS) with implantation of IOLs (acrylic foldable, heparin surface modified and non-heparin surface modified). Detailed history, ophthalmological examination, general physical examination was carried out.

Preoperative visual acuity measurement, pupillary examination, slit lamp examination (for any edema, inflammation and type of cataract), fundus examination, IOP measurement, syringing, blood pressure, urine for albumin and sugar, blood both fasting and post-prandial sugar, IOL power calculation. Very dense cataract, no biomicroscopically detectable diabetic retinopathy, age $<50$ years, previous laser treatment, any other ocular disease, use of systemic or local anti-inflammatory drugs, pre-existing uveitis, corneal dysgenesis, dystrophies, or opacities were excluded from the study.

Surgery was performed by senior ophthalmic surgeons under local anesthesia. Mydriasis was achieved with tropicamide and phenylephrine drops instilled 3 times every 10-15 minutes before surgery. Povidine iodine $(5 \%)$ was instilled in the conjunctival sac.

For performing Phacoemulsification two types of incision was made scleral, clear corneal and was grooved up to $1 \mathrm{~mm}$ clear cornea using crescent knife. Entry was made with 3.2 keratome. Capsulorrhexis was done followed by hydrodissection and hydrodelineation. Mainly divide and conquer technique was used, by using irrigation/aspiration port epinucleus and cortical matter was removed followed by lens implantation and closure of wound. For performing SICS $5.5 \mathrm{~mm}$ straight incision was given 2 to $2.5 \mathrm{~mm}$ behind the limbus and $1 \mathrm{~mm}$ clear corneal tunnel was made. Entry was made by 3.2 keratome, anterior chamber maintainer was introduced at 6 o'clock position. Capsulotomy with 26G bent needle was done followed by hydrodissection and nucleus prolapse into $\mathrm{AC}$ and then extraction of nucleus by using wire Vectis, phaco-sandwich technique, bluementhal technique. Simcoe cannula used for cortical washing. Rigid PMMA IOL was implanted.

Post operatively patients were discharged with advice to use topical antibiotic ciprofloxacin e/d QID, steroid prednisolone e/d QID, flurbiprofen e/d QID, mydriatic tropicamide with phenylephrine e/d HS was instructed to review every week for 4 weeks and then monthly for 6 months. On each visit slit lamp examination, vision assessment and ophthalmoscopy done. FFA at 1 week and 6 month post operatively. Final visual acuity and prescription of glasses after 6 weeks post operatively done.

\section{Results}

Majority of patients of age group 51-60 and most of them were females. 15 patients from each group divided according to the status of DR as BDR, Pre proliferative, Proliferative and DR with maculopathy. Both surgical procedure was employed for every stage of retinopathy and three different types of IOL is implanted. Main per-operative complication were intra-operative miosis and pigment dispersion. Striate keratopathy mainly seen in patients with phacoemulsification, no dependence upon the stage of retinopathy, but iridocyclitis was the main immediate post-operative complication. Postoperative uveitis seen mainly in PDR patients with PMMA IOLs (50\%). Acrylic and HSM IOLs had almost equal incidence of uveitis. Out of total 60 patients, in majority eyes $(86 \%)$ visual acuity improved by 2 or more lines on Snellen's chart. $21.6 \%$ patients had visual acuity batter than 6/12. None of the patients with PDR or DR with maculopathy had VA 6/12 or better. Incidence of cystoid macular edema increased as the stage of retinopathy increased. Mainly in PDR patients, CME was seen and that too when procedure employed was phacoemulsification. Posterior capsular opacification was mainly in patients with PMMA lens implantation. Though one case with PCO was also reported in acrylic IOL. After 6 months of follow up, PCIOL deposits was main complication seen. Two patients with vitreous hemorrhage also reported after 6 months of operation. Progression of retinopathy was seen in seven cases, BDR converting to Pre-PDR and Pre-PDR into PDR within 3 months post operatively.

\section{Discussion}

Cataract is a recognized complication of diabetes and it has been estimated that upto $20 \%$ of all cataract surgery is performed on diabetic patients. The 1997 guidelines of 
Table 1: Operative procedures performed and type of IOL implanted in four status of DR patients.

\begin{tabular}{lcccc}
\hline & BDR & Pre-PDR & PDR & DR with maculopathy \\
Operative procedure: & 9 & 11 & 12 & 11 \\
SICS & 6 & 4 & 3 & 4 \\
PHACO & & & & 5 \\
TYPE OF IOL: & & & 5 & 4 \\
SICS- & 4 & 4 & 4 & 2 \\
PMMA & 3 & 5 & 3 & 1 \\
HSM & 2 & 2 & 1 & 1 \\
ACRYLIC & & & 1 \\
PHACO- & 1 & 1 & 1 \\
PMMA & 2 & 1 & \\
HSM & 3 & 2 & & \\
ACRYLIC & & & \\
\hline
\end{tabular}

Table 2: Incidence of uveitis among cases

\begin{tabular}{lcccc}
\hline Incidence of uveitis & BDR & Pre-proliferative & Proliferative & DR with maculopathy \\
PMMA & 0 & 2 & 3 & 2 \\
HSM & 0 & 0 & 1 & 0 \\
ACRYLIC & 0 & 1 & 0 & 0
\end{tabular}

Table 3: Postoperative best corrected visual acuity

\begin{tabular}{|c|c|c|c|c|}
\hline BCVA & BDR & Pre-proliferative & Proliferative & DR with maculopathy \\
\hline \multicolumn{5}{|l|}{ SICS } \\
\hline$<6 / 60$ & 1 & 0 & 4 & 3 \\
\hline $6 / 60-6 / 36$ & 1 & 5 & 7 & 6 \\
\hline $6 / 36-6 / 18$ & 2 & 4 & 1 & 2 \\
\hline$>6 / 12$ & 5 & 2 & 0 & 0 \\
\hline \multicolumn{5}{|l|}{ PHACO } \\
\hline$<6 / 60$ & 0 & 0 & 4 & 3 \\
\hline $6 / 60-6 / 36$ & 0 & 1 & 7 & 6 \\
\hline $6 / 36-6 / 18$ & 2 & 1 & 1 & 2 \\
\hline$>6 / 12$ & 4 & 2 & 0 & 0 \\
\hline
\end{tabular}

Table 4: Incidence of CSME

\begin{tabular}{lccc}
\hline Incidence of CSME & BDR & Pre-proliferative & Proliferative \\
SICS & 2 & 4 & 8 \\
PHACO & 1 & 4 & 6 \\
Progression of DR & 3 & 4 & 0 \\
\hline
\end{tabular}

the Royal College of Ophthalmologists states that all form of diabetic retinopathy may become more severe following cataract surgery.

In this study we took patients with DR of various grades. Fifteen patients each from various grades, underwent cataract surgery using non-operated fellow eye as control. Different surgical techniques were employed and variety of IOLs implanted. Patients without diabetic retinopathy were excluded. The patients with PDR and particularly DR with maculopathy fared less. However, patients with maculopathy or severe retinopathy had valuable visual impairment after surgery and clear optical axis allowing adequate laser photocoagulation. In the study, visual acuity after cataract surgery was improved by two or more lines on snellen's chart in majority of cases (86\%). $21.6 \%$ patients had visual acuity better than 6/12.

In our study, total of 60 patients were taken, divided into four equal groups of 15 patients each as BDR, Pre-PDR, PDR, DR with maculopathy. Out of 15 patients with BDR 13 got visual acuity $>6 / 12(86 \%)$. 5 patient had visual acuity 6/18-6/36, 6 had visual acuity 6/36-6/60, in pre-PDR group of 15 patient. Out of 15 patients with PDR, 1 patient had visual acuity of 6/18-6/36, nine had VA of 6/36-6/60 and 5 had VA of $<6 / 60$. Patients with DR with maculopathy majority of them attend VA from $6 / 36$ to $<6 / 60$.

Similar results were reported by Dowler et $\mathrm{al}^{1}$ who in their metanalysis studied that NPDR with no maculopathy patients attain VA better than $6 / 12$ in approximately 


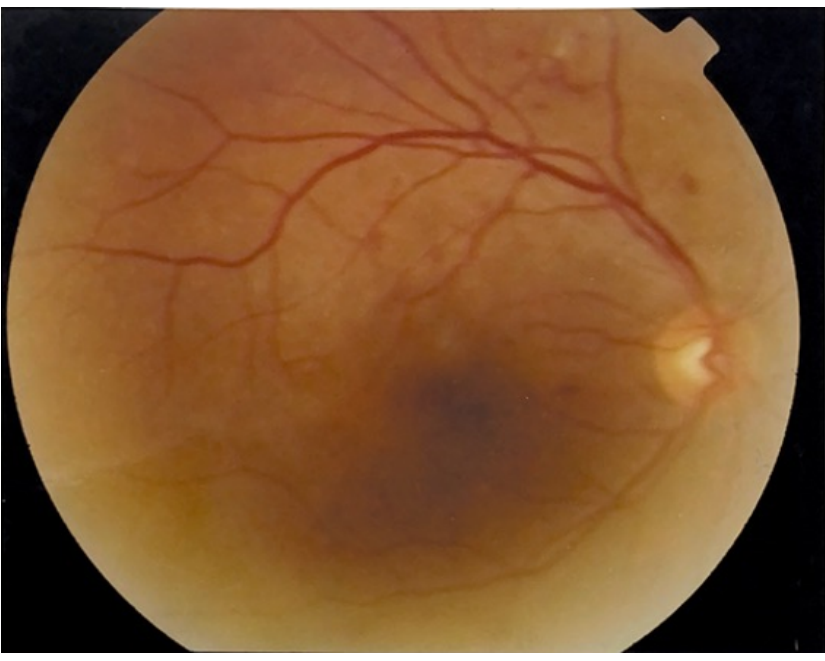

Fig. 1: NPDR in a patient with background retinopathy six months of follow up after cataract surgery

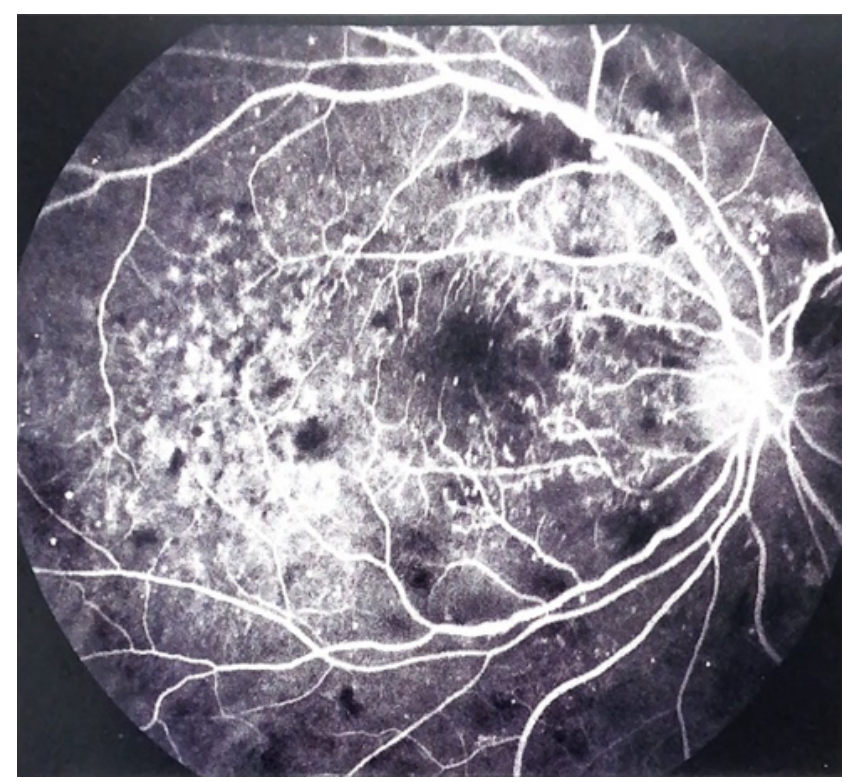

Fig. 2: PDR in a patient with NPDR six months of follow up after cataract surgery

$80 \%$ cases which is close to our study. Cunliffe et $\mathrm{al}^{2}$ retrospectively studied patients of BDR without maculopathy attaining VA better than $6 / 12$ following cataract surgery approximately $56.5 \%$.

Key point remains the same i.e., post operatively visual impairment simply reflects the pre-operative retinal and macular status in diabetics.

Zaczek et $\mathrm{al}^{3}$ prospectively studied the visual acuity after phacoemulsification. 41 diabetic eye $(79 \%)$ achieved VA of $6 / 12$ or better and 11 eyes $(21 \%)$ had final VA lower than 6/12. Eyes with mild to moderate DR and CSME 1 week post-operatively had a lower final VA than those without

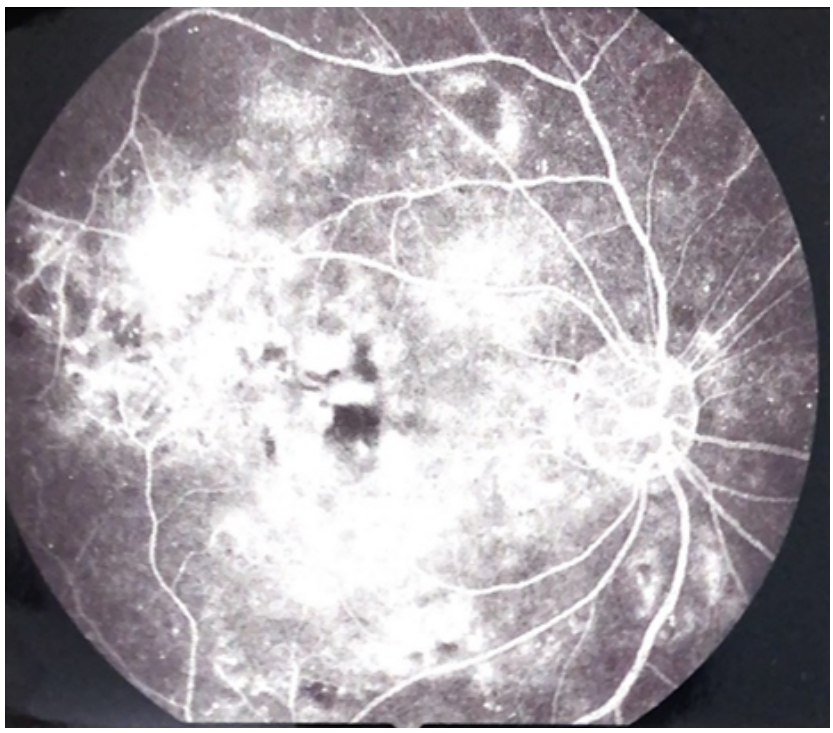

Fig. 3: PDR in a patient with NPDR six months of follow up after cataract surgery

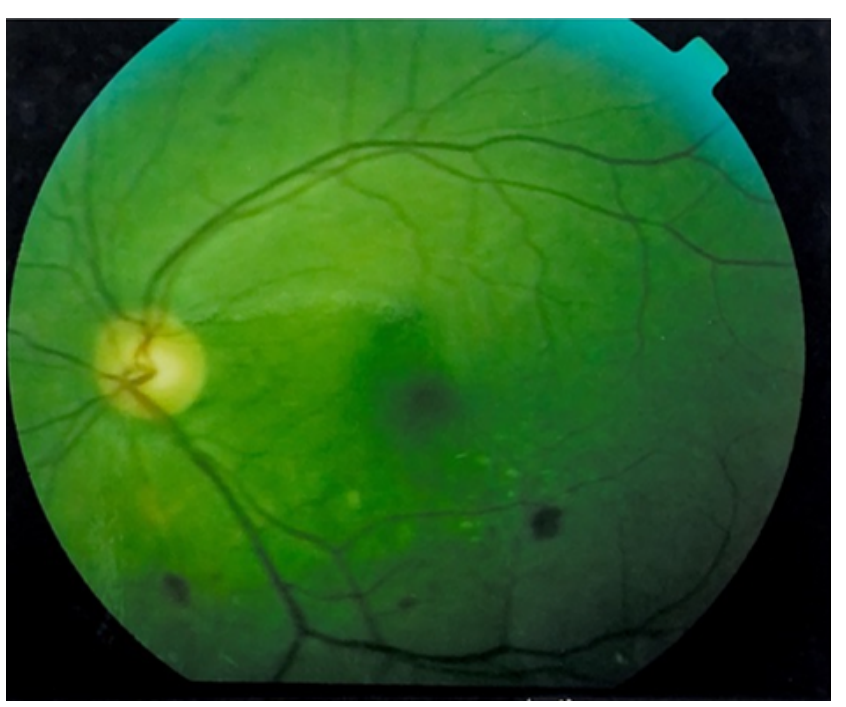

Fig. 4: NPDR in a patient with background retinopathy six months of follow up after cataract surgery

\section{CSME.}

So, final VA depends upon the initial status of retinopathy and not on mode of surgery employed or type of IOL implanted, also VA is poor in patients with PDR, or DR with maculopathy.

Our prospective study showed pigment dispersion and inter-operative miosis as major pre-operative complications. Among post-operative complications striate keratopathy, iridocyclitis (26\%) mainly in pre-PDR and PDR, deposits on IOL. No difference was noted in inflammation with use of different types of IOLs. 
Studies of cunliffe et $\mathrm{al}^{2}$ and zaczek et $\mathrm{al}^{3}$ have shown that diabetic eye have more complications after cataract surgery than non-diabetic eyes, particularly more pronounced post-operative inflammation.

We implanted HSM IOL in all groups of DR, 5 each in BDR, PDR, DR with maculopathy and 6 in Pre-PDR. Postoperative inflammation was less compared to PMMA IOL and equivalent to acrylic IOL in phacoemulsification. This result was equivalent to those given by katharina krepler et $\mathrm{al}^{4}$ gatinel D et al. ${ }^{5}$

Cunliffe et $\mathrm{al}^{2}$ in his retrospective study shows that rubeosis iridis and neovascular glaucoma was common in diabetics while no case were reported in non-diabetic control.

Hykin et $\mathrm{al}^{6}$ have reported that miosis and neovascular glaucoma is more common in PDR group as compared to no cases in BDR group.

In our study 2 out of 15 cases of PDR developed vitreous hemorrhage and none developed neovascular glaucoma. As compared to Cunliffe et $\mathrm{al}^{2}$ study, $6.4 \%$ cases of vitreous hemorrhage, our study reported $3.3 \%$ cases of vitreous hemorrhage.

Progression of diabetic retinopathy was found in 7 cases out of 60 patients underwent cataract surgery. While study by Anna Zaczek et $\mathrm{al}^{3}$ and D. Squirrel et $\mathrm{al}^{7}$ showed progression of diabetic retinopathy in $21 \%$ and $20 \%$ eyes respectively.

\section{Conclusion}

The study was conducted to evaluate the visual outcome, the incidence of postoperative inflammation, CME and progression of retinopathy following cataract surgery (SICS and Phacoemulsification) in patients with various grades of diabetic retinopathy with implantation of various IOLs. A total of 60 patients with different grade of diabetic retinopathy was included. Visual outcome improves in majority of eyes and was not dependent upon the surgical technique employed or the type of IOL implanted. Visual acuity $>6 / 12$ in $22 \%$ of cases, in BDR ( 9 out of $15,60 \%$ ), Pre PDR (4 out of 15, 26\%). PDR and DR with maculopathy did not attain visual acuity of $>6 / 12$. Post- operative uveitis was maximum with PDR. Majority of patients with PDR developed cystoid macular edema. Progression of retinopathy and maculopathy represented the natural history of disease not upon the surgical technique employed.

\section{Conflicts of Interest}

All contributing authors declare no conflicts of interest.

\section{Source of Funding}

None.

\section{References}

1. Dowler JGF, Hykin PG, Lightman S. Visual acuity following extracapsular cataract extraction in diabetes: A meta-analysis. Eye 1995;9:313-7.

2. Cunliffe IA, Flanagan DW, George ND, Aggarwaal RJ, Moore AT. Extracapsular cataract surgery with lens implantation in diabetics with and without proliferative retinopathy. Br J Ophthalmol. 1991;75(1):912. do1:10.1136/bjo.75.1.9.

3. Anna Z. Olivestedt Goran et al Visual outcome after phacoemulsification and IOL implantation in diabetic patients Br J Ophthalmol. 1999;p. 1036-41.

4. Katharina K, Eva R. Derbolav Agnes et al Inflammation after phacoemulsification in diabetic retinopathy. Foldable acrylic versus heparin surface modified polymethyl methacrylate intraocular lenses. J Cataract Refract Surg. 2001;27:233-8.

5. Gatinel D, Lebrun T, Toumelin PL, Chaine G. Aqueous flare induced by heparin-surface-modified poly(methyl methacrylate) and acrylic lenses implanted through the same-size incision in patients with diabetes. J Cataract Refractive Surg. 2001;27(6):855-60. 101:101016/s0886 B350(00)00814-2.

6. Philip GH, Richard M, Gregron. Extracapsular cataract extraction in proliferative diabetic retinopathy. Ophthalmol. 1993;100:394-9.

7. Squirrell D. A prospective, case controlled study of the natural history of diabetic retinopathy and maculopathy after uncomplicated phacoemulsification cataract surgery in patients with type 2 diabetes. Br J Ophthalmol. 2002;86(5):565-71. do1:10.1136/bjo.86.5.565.

\section{Author biography}

Aparajita Chaudhary, Professor

Smriti Kumari, IIIrd Year Junior Resident

Cite this article: Chaudhary A, Kumari S. A Comparative study of post-operative results with different grades of diabetic retinopathy after phacoemulsification and small incision cataract surgery (SICS) [with various types of IOLs]. IP Int J Ocul Oncol Oculoplasty 2020;6(4):232-236. 